\title{
INVESTIGACIÓN SOBRE VALORES GENERALES EN UN GRUPO DE ADOLESCENTES DE SANTO DOMINGO.
}

RESUMEN.

Francia Reynoso*

El siguiente artículo resume los resultados de una investigación realizada con 300 adoiescentes de distintos estratos sociales de la ciudad de Santo Domingo.

La metodología ha sido innovadora en cuanto los propios adolescentes contribuyeron en su construcción así como en la definición de las cualidades, principios y elementos negativos que consideraron como válidas para ser consideradas como valores

En la misma se evidencia el predominio de una selección de valores positivos sobre otros de signo negativo que son rechazados en términos generales.

Las diferencias de género no aparecen como elementos diferenciadores en las formas de valorar entre este grupo de adolescentes.

Los resultados solo son válidos para el grupo estudiado y hablan de su manera de estimar, más no sirven de referente para ubicarlos como parte de sus prácticas de vida.

Aparece como reto dar continuidad a este tipo de investigaciones relacionando valores y prácticas.

PALABRAS ClAVES:

Valores, adolescencia

\section{INTRODUCCIÓN}

Esta investigación fue realizada en un grupo de adolescentes medios de la ciudad de Santo Domingo. La muestra fue de 300 adolescentes de distintos estratos sociales con el interés de cono-

(") Equipo de Investigación CENINBIO - INTEC 
cer cuáles eran los valores generales predominantes y qué era lo que ellos valoraban como importante en su mundo de participación.

Para realizar la investigación desarrollamos la siguiente metodología:

\section{METODOLOGÍA}

El primer paso metodológico consistió en reunir un grupo de adolescentes a los cuales se les solicitó que hicieran una lista de los que consideraban que eran los principales valores en su vidas de adolescentes. No se dio ningún insumo previo como definiciones de valores y solo nos limitamos a señalar que consideraríamos como valor lo que ellos estimaran como tal.

De esta reunión salió una lista con 50 valores de distintos órdenes los cuales luego procedieron a definir y a la que nosotros colocamos una escala de importancia.

La técnica utilizada para nuestra investigación fue de carácter cualitativo en sus inicios. Para ello introdujimos la técnica de grupos focales, que consiste en una reunión de grupos de iguales o grupos de pares, con los cuales a través de un proceso de interacción, discusión y elaboración de conceptos, sobre la temática de valores, pudimos precisar sobre sus percepciones y definiciones .

En esas primeras sesiones grupales constituimos los grupos con estudiantes adolescentes entre 14 y 17 años, con la tarea de discutir, ampliar y elaborar las definiciones de los valores ya listados. Las sesiones estaban realizadas por un moderador y un relator.

Después de tener definidos los valores de nuestra investigación, se procedió a la administración de la escala a los grupos señalados anteriormente que participaron en la investigación. La escala de importancia fue la siguiente: 


\section{0 - 2 Nada importante \\ 3-4 Importante \\ 5 - 6 Muy importante}

\section{RESULTADOS}

El estudio se realizó con 300 adolescentes entre los 14 y 17 años, de diferentes estratos sociales de la ciudad de Santo Domingo de los cuales eliminamos 38 que por distintas razones no cualificaron. De este universo $131(50 \%)$ eran del sexo masculino y $131(50 \%)$ del sexo femenino.

Para nuestro estudio nos interesó inicialmente analizar la presentación de la importancia de los valores sin tomar en cuenta el sexo y en una segunda etapa hacer la correlación según sexo para poder medir diferencias de género.

En esta escala del 0-2 significa que estos valores no le interesan para nada, 3-4 les son importantes y de 5-6 muy importantes. Es necesario recordar que el concepto de valor utilizado está referido a lo que les interesa o importa y no al concepto moral del valor, de manera que lo que podría estar categorizado socialmente como un disvalor o valor negativo aparecerá como valor si así lo han reconocido en su valoración.

A continuación exponemos la frecuencia de aparición de los valores y la jerarquía en la importancia que los adolescentes le asignaron (ver Cuadros N. ${ }^{\circ} \mathrm{s} 1$ - 7).

En esta distribución de frecuencias podemos ver como los y las adolescentes establecen un orden y una jerarquía axiológica según sus formas de comprender el valor, definido desde lo que les interesa y en grados de importancia.

En esta primera presentación aparecen todos los valores por ellos y ellas identificados como tales sin hacer distinción de alcance, pero determinando claramente orden y jerarquía. 


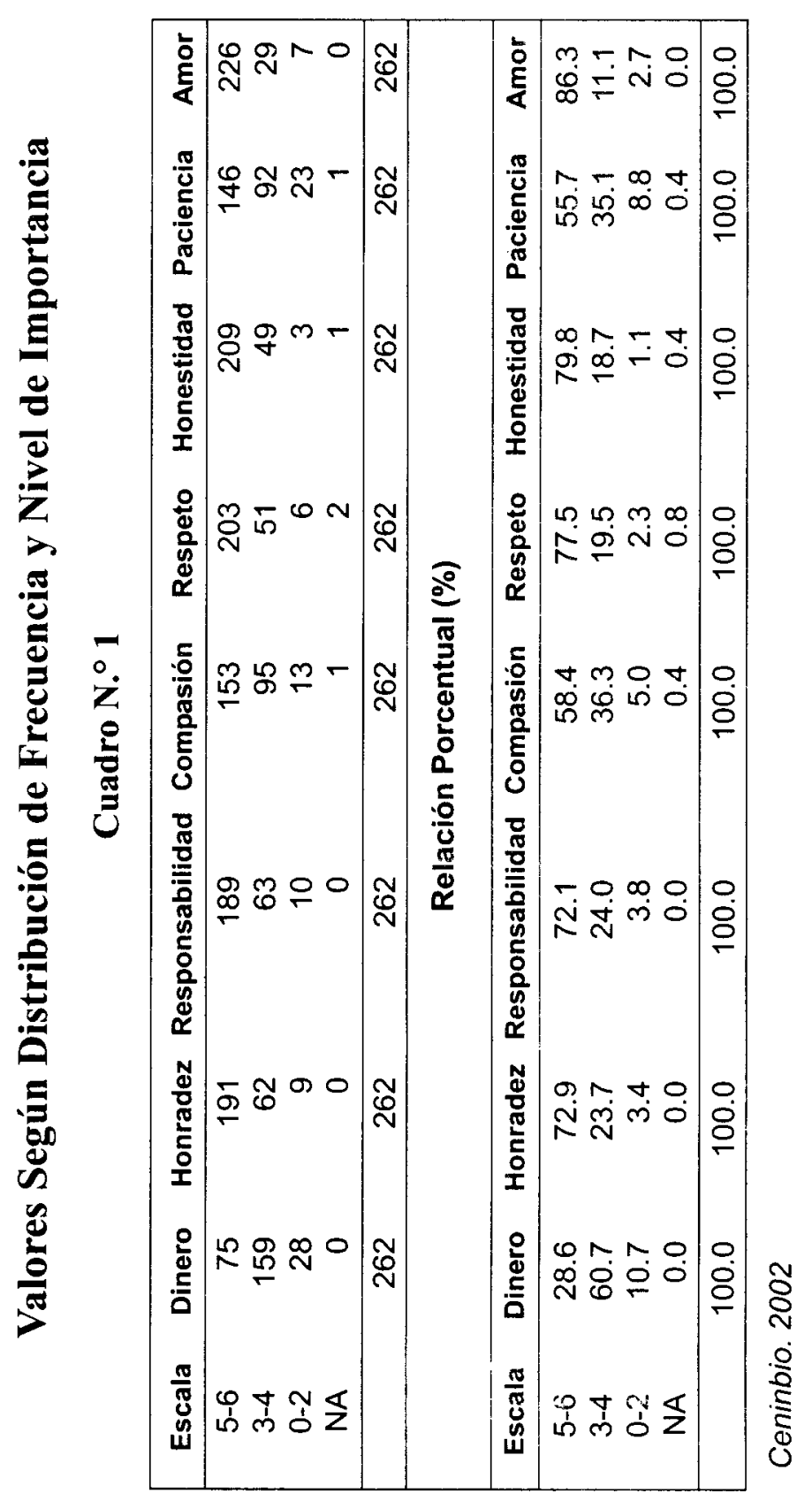

611 


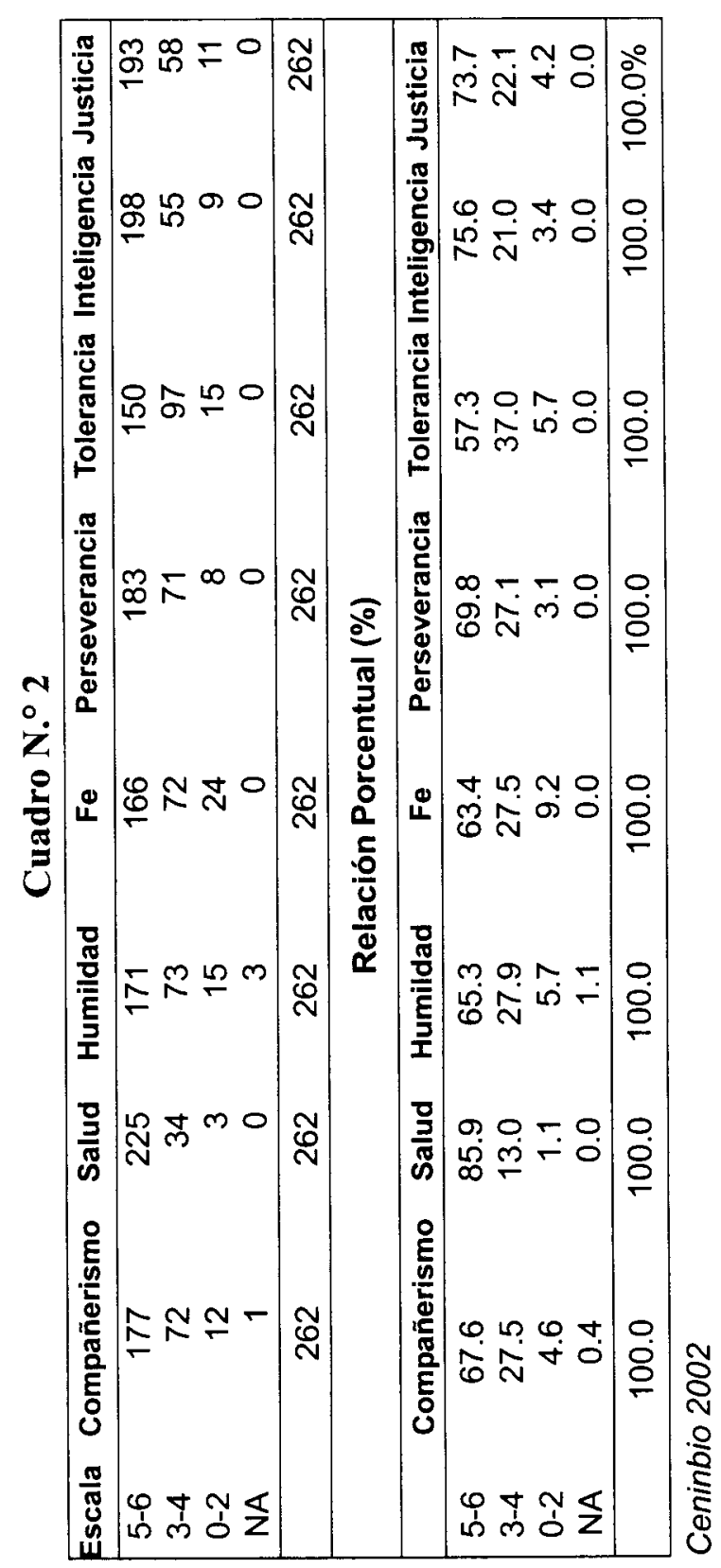

612 


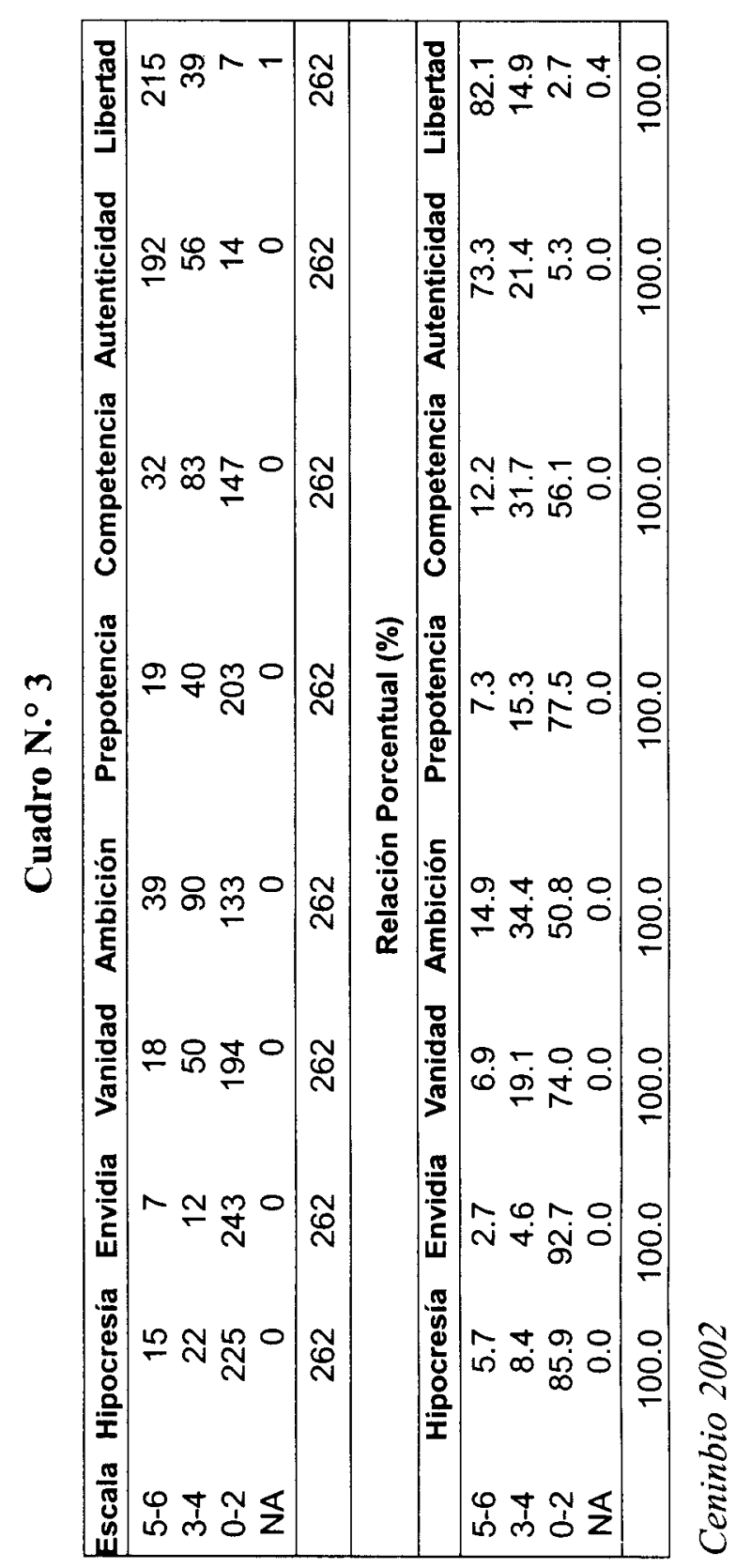




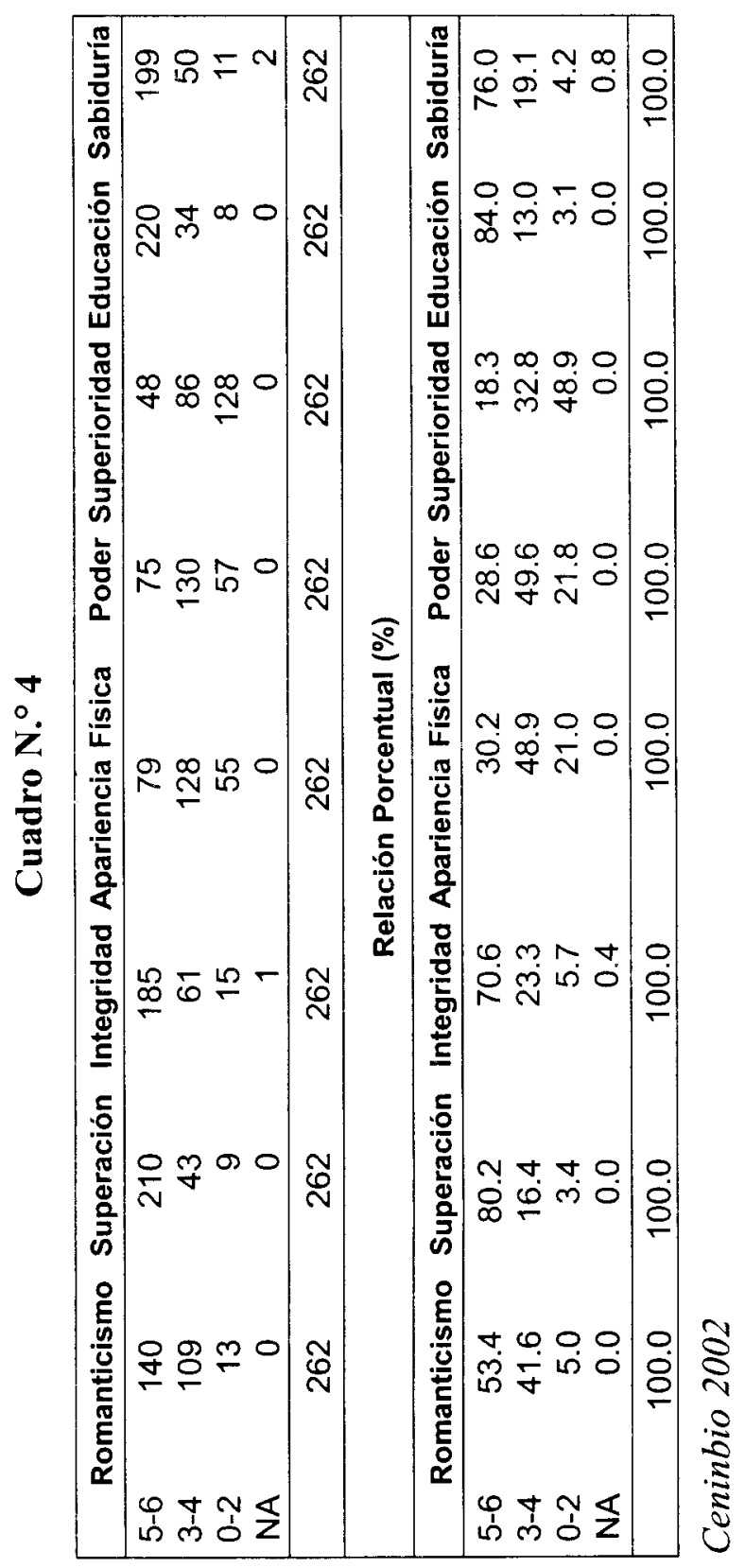

614 


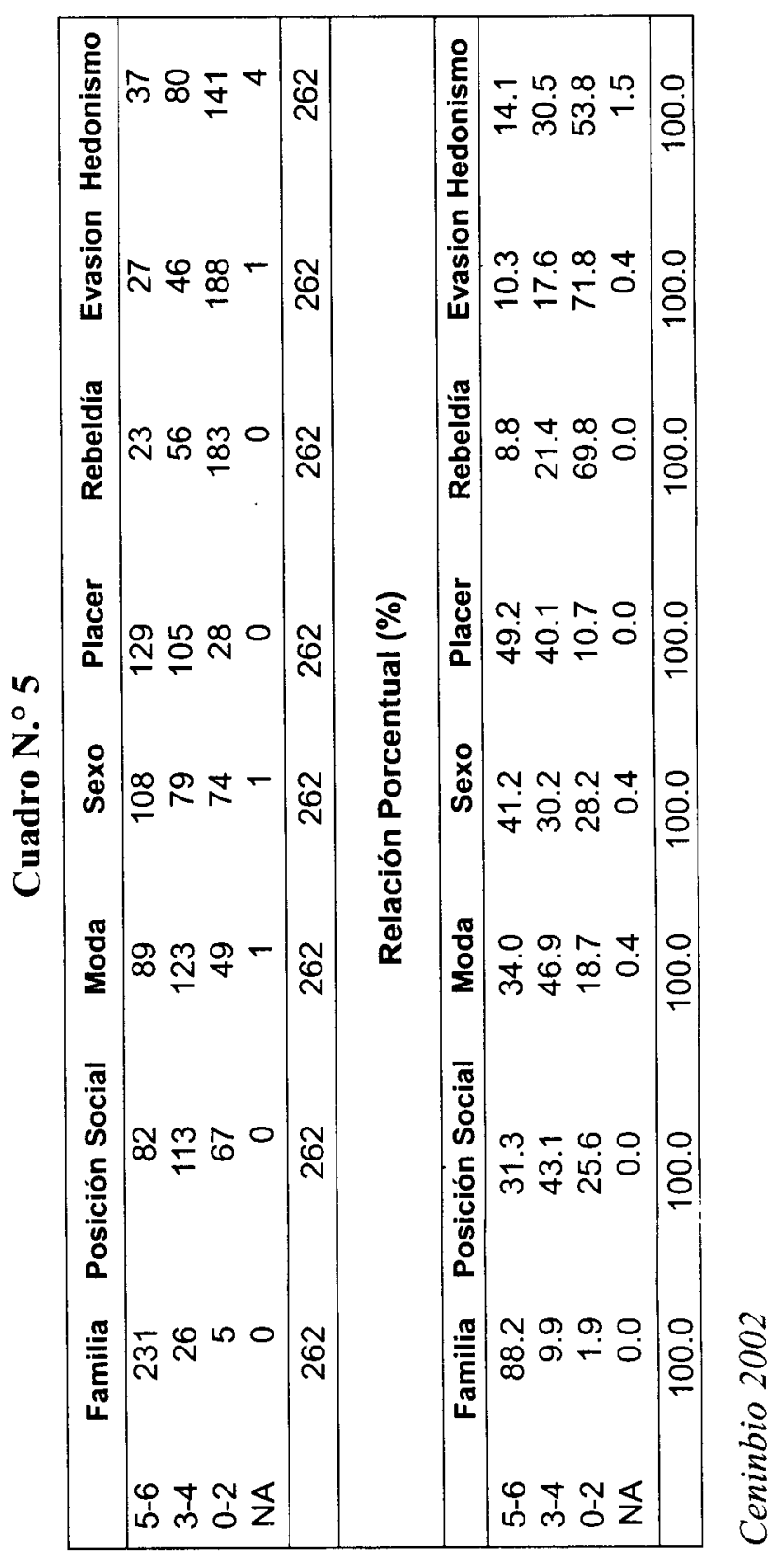




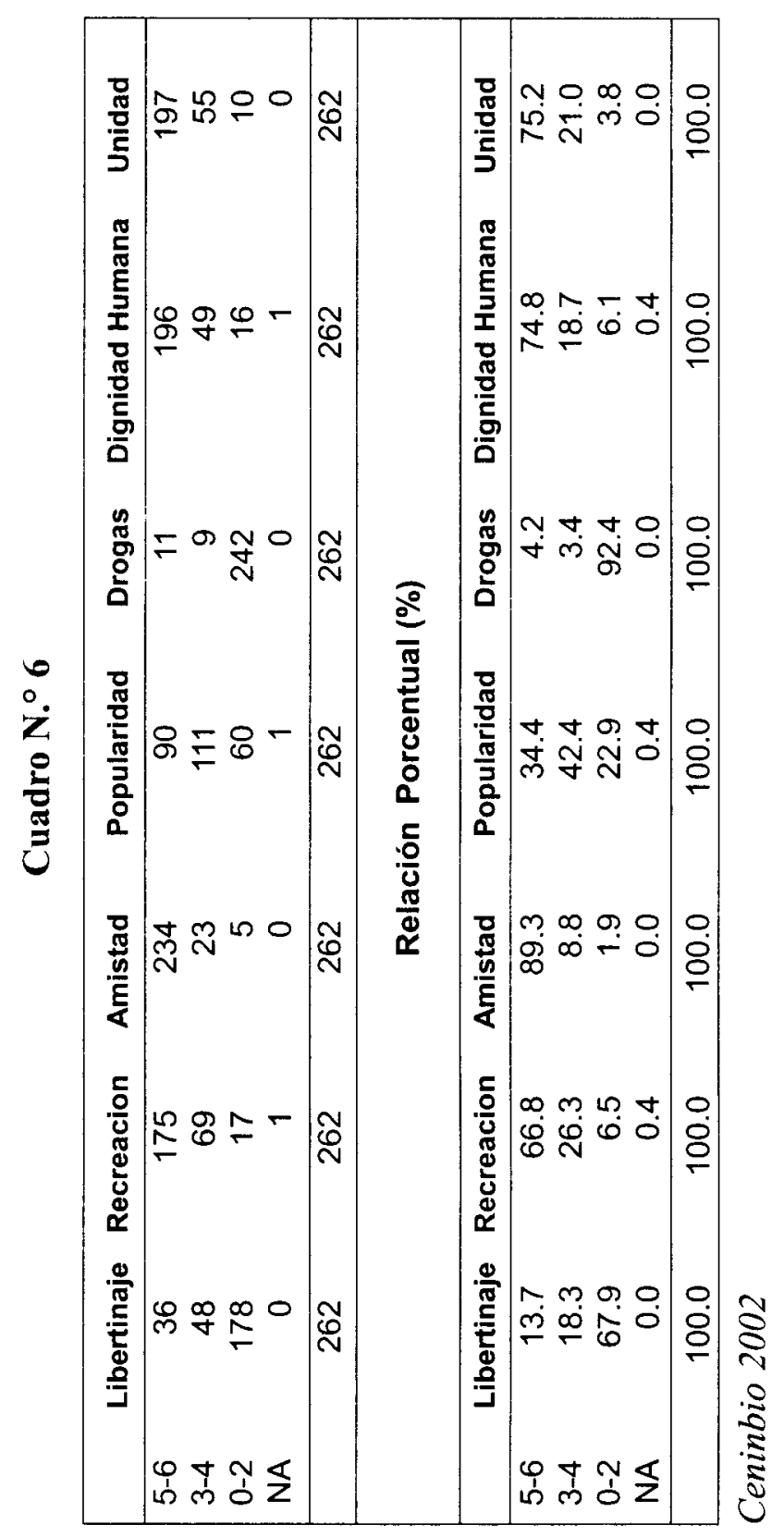

616 


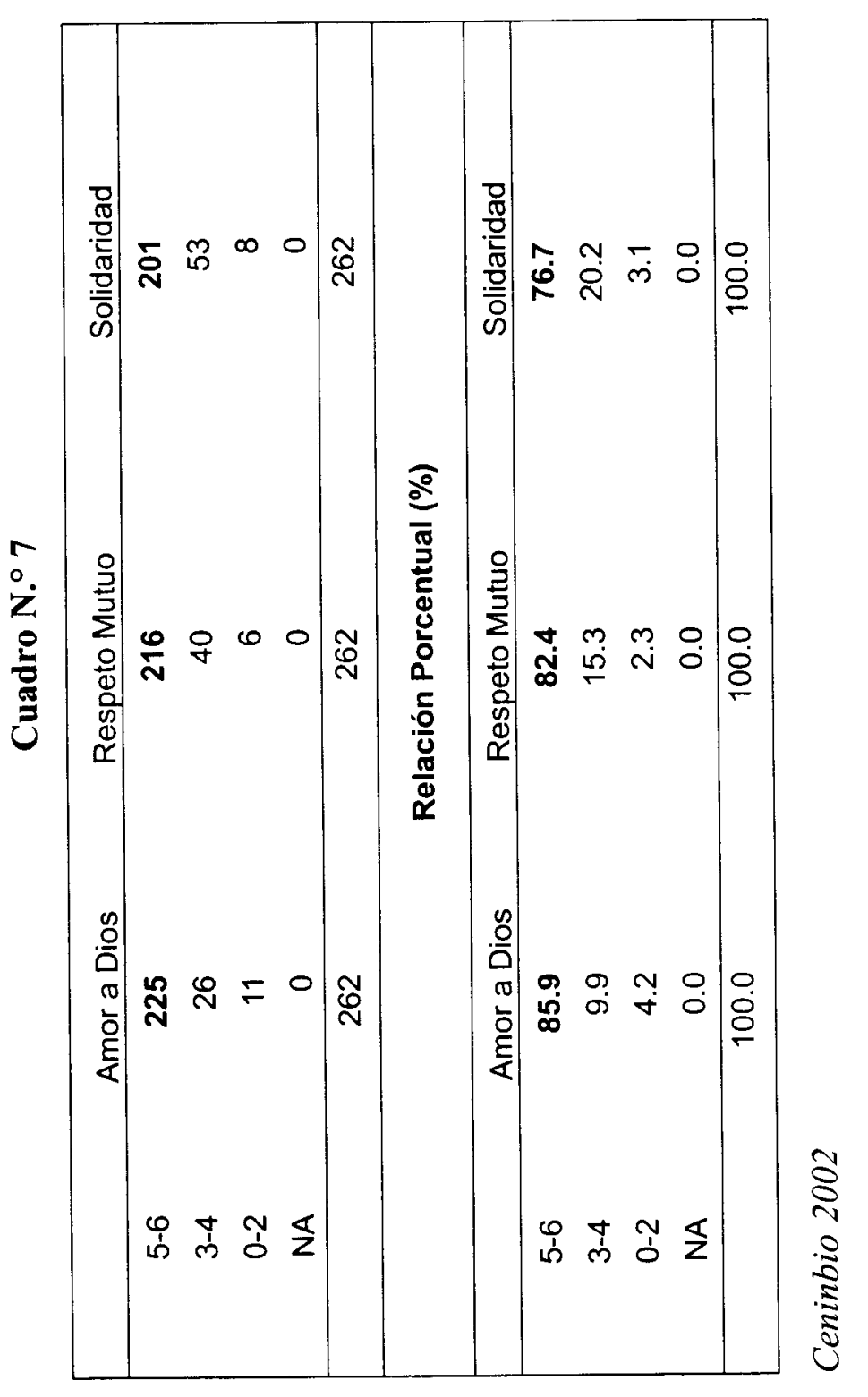


En los planteamientos clásicos sobre el valor Scheler y Hartmann hicieron grandes aportes y conceptualizaciones que aquí no vamos a debatir, sin embargo retomamos de sus posiciones la idea de que cada sistema de valores incluye una jerarquía y un ordenamiento. Ellos lo predeterminaron dando pautas sobre valores superiores e inferiores. En esta investigación los adolescentes hacen un planteo diferente en cuanto son los que deciden a qué llamarán valor y parten de la premisa que les entregamos de que "valor es lo que vale," de ahí que hayan incluido como tales elementos que anteriormente eran considerados como disvalores .

Las mayores votaciones la obtuvieron valores como la amistad con un $89.3 \%$ en la categoría de "muy importante" o la negación de drogas, considerando en un $92.4 \%$ que esto corresponde a "nada importante" en sus vidas.

En términos generales la polaridad positiva de los valores obtuvieron la más alta puntuación lo que indica su identificación con ellos. Valores como "dinero" y "popularidad" que guardan una cierta ambigüedad en su interpretación pasaron a un segundo lugar, lo cual entendemos como normal sobre todo porque no dimos interpretación de parte del equipo de investigadores.

El aporte innovador de esta metodología es que son los propios jóvenes quienes deciden la definición de lo que consideran un valor sin la intervención premoralizante del investigador que tradicionalmente coloca una lista de valores preconstruidos y validados por la moral adulta.

A seguidas hicimos una subclasificación de los valores entregados por el estudio reconociendo cuatro categorías:

1. Físicos. Incluye salud, apariencia fisica e interés por el sexo. En este acápite se reconocen valores vinculados al cuerpo y sus intereses.

2. Valores positivos. La visión enfatizada por Frondizi sobre la bipolaridad de los valores es recogida en 
nuestro estudio clasificando según el signo de la polaridad en positivos y negativos

Estos valores positivos pueden ser considerados como virtudes. Hemos ubicado en este lugar aquellos valores cuyas prácticas conducen a la felicidad, correlacionándola con la ética de las virtudes que asumen que el ser humano está orientado por su naturaleza a la felicidad y que determinadas prácticas dirigen a su consecución,

"la contemplación de la verdad ideal es el ideal superior de vida virtuosa. El bienestar de toda la sociedad es lo que debe orientar las relaciones entre sus miembros" 1

3. Valores negativos. Son los que desdicen o niegan a los positivos. Son los componentes opuestos al polo positivo que representa a su vez la moralidad del valor, independientemente de que no estuviéramos valorando ese componente.

\subsection{Aspectos Relacionados al Cuerpo}

Fisicos

\begin{tabular}{|cccc|}
\hline Escala & Salud & Apariencia & Sexo (interés) \\
\hline $5-6$ & $\mathbf{2 2 5}$ & 79 & $\mathbf{1 0 8}$ \\
$3-4$ & 34 & $\mathbf{1 2 8}$ & 79 \\
$0-2$ & 3 & 55 & 74 \\
NA & 0 & 0 & 1 \\
\hline Total & 262 & 262 & 262 \\
\hline
\end{tabular}

Según este cuadro les resulta más importante la salud que cualquier otro de los valores de esta categoría siguiéndole el inLuis José González Alvarez. Etica latinoamericana. Universidad Santo Tomas Bogo-
tá 1994. P29 
terés por el sexo y en tercer lugar la apariencia física como "importante".

El que la salud aparezca con tan alta votación es un signo de mucha importancia dado que una de las características de la adolescencia media es el desarrollo del sentimiento de invulnerabilidad donde los adolescentes de ese estadio se consideran invencibles y ponen en dudas el que a ellos les pueda suceder algo.

Si aparece la salud como un elemento de tanta importancia podemos afirmar que en esa población se ha logrado permear los mensajes de prevención y conservación del estado de salud. Es una estimación que no necesariamente habla a favor de sus prácticas de preservación de la salud.

En la adolescencia media se puede reconocer como una conducta esperada la valoración de los aspectos relacionados a la salud y la apariencia por pertenecer al espacio de lo físico y la belleza relacionada con el aspecto. Es el tiempo del culto al cuerpo.

La sexualidad por su parte hace su pico más alto en tanto se reconoce y crece el interés por el sexo opuesto

La vinculación entre el proceso de independizarse, de acoger el grupo de pares como el centro de su interés y relacionarse con el sexo opuesto interesados por ello, justifican o pueden explicar la aparición de estos tres valores asociados al cuerpo y podemos considerarlo como conductas esperadas con un sustrato axiológico de esta magnitud porque es lo que sustenta a los cambios sicológicos, físicos y sociales de esa etapa.

\subsection{Aspectos Relacionados a las Virtudes}

Para Frondizi los valores son bipolares ya que todos tienen un componente positivo y otro negativo como parte de la unidad de todo valor $y$ las personas asumimos en libertad o por aprendizaje, inducción u otro mecanismo uno de los dos extremos identificándonos con el polo positivo o el negativo del valor, de ahí 


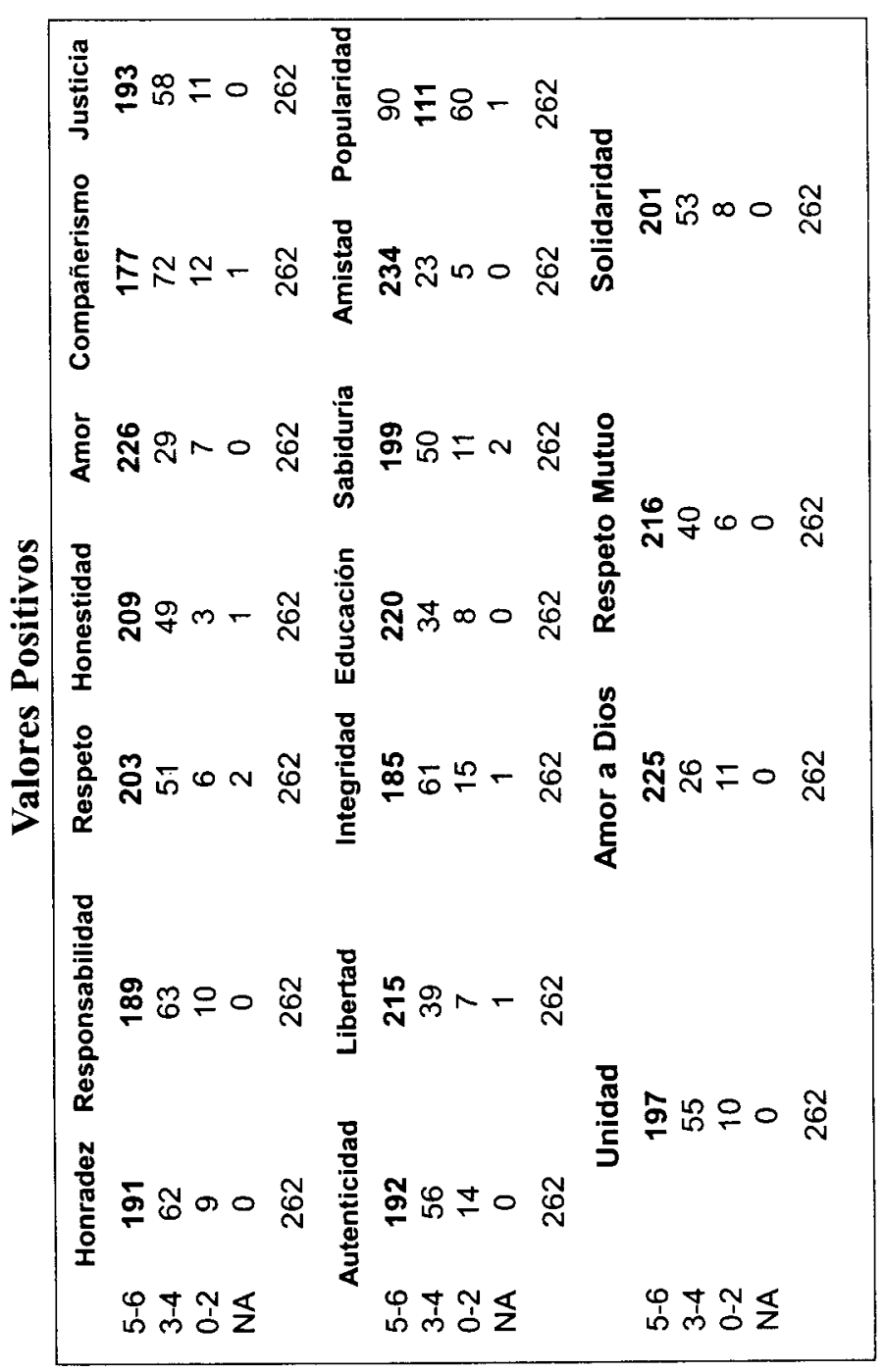


que existirá una alta posibilidad de que nuestras acciones se orienten en correspondencia con la posición asumida.

En estos cuadros podemos observar que todos los valores positivos o polos positivos de esos valores fueron señalados como los más votados en la categoría de "muy importantes" en su vida, excepto la popularidad que para ellos es"ser conocidos por varias personas" y cae en la categoria de "importantes".

Si bien es cierto que estos resultados solo evidencian estimaciones y valoraciones y no comportamientos, no es menos cierto que son un componente esencial para la implementación de estrategias educativas para orientar hacia la asunción de prácticas axiológicamente positivas.

La tarea educativa no tiene más opción que trabajar por clarificar cuáles son los polos positivos de los valores y trabajar por orientar hacía su construcción. La construcción del polo positivo es a su vez trabajar por construir el valor moral ya que el polo positivo corresponde a la moralidad del valor con el beneficio de que con esta metodología son los propios adolescentes quienes lo definen y quienes lo posicionan en orden y jerarquía.

Como ya existen en la valoración máxima, dan pistas de la posibilidad de establecer estrategias educativas que partan de este punto con el interés de reforzar la construcción de valores positivos.

\subsection{Aspectos Relacionados al Disvalor}

Si entendemos estos valores como polos negativos debemos entender que su opuesto existe, para hipocresía podría ser honestidad y lo mismo para cada uno de los señalados en cada cuadro.

El sentido de identificar en los adolescentes sus estimaciones para los polos positivos y negativos nos demuestran con números que sus valoraciones niegan el disvalor ya que las mayores 


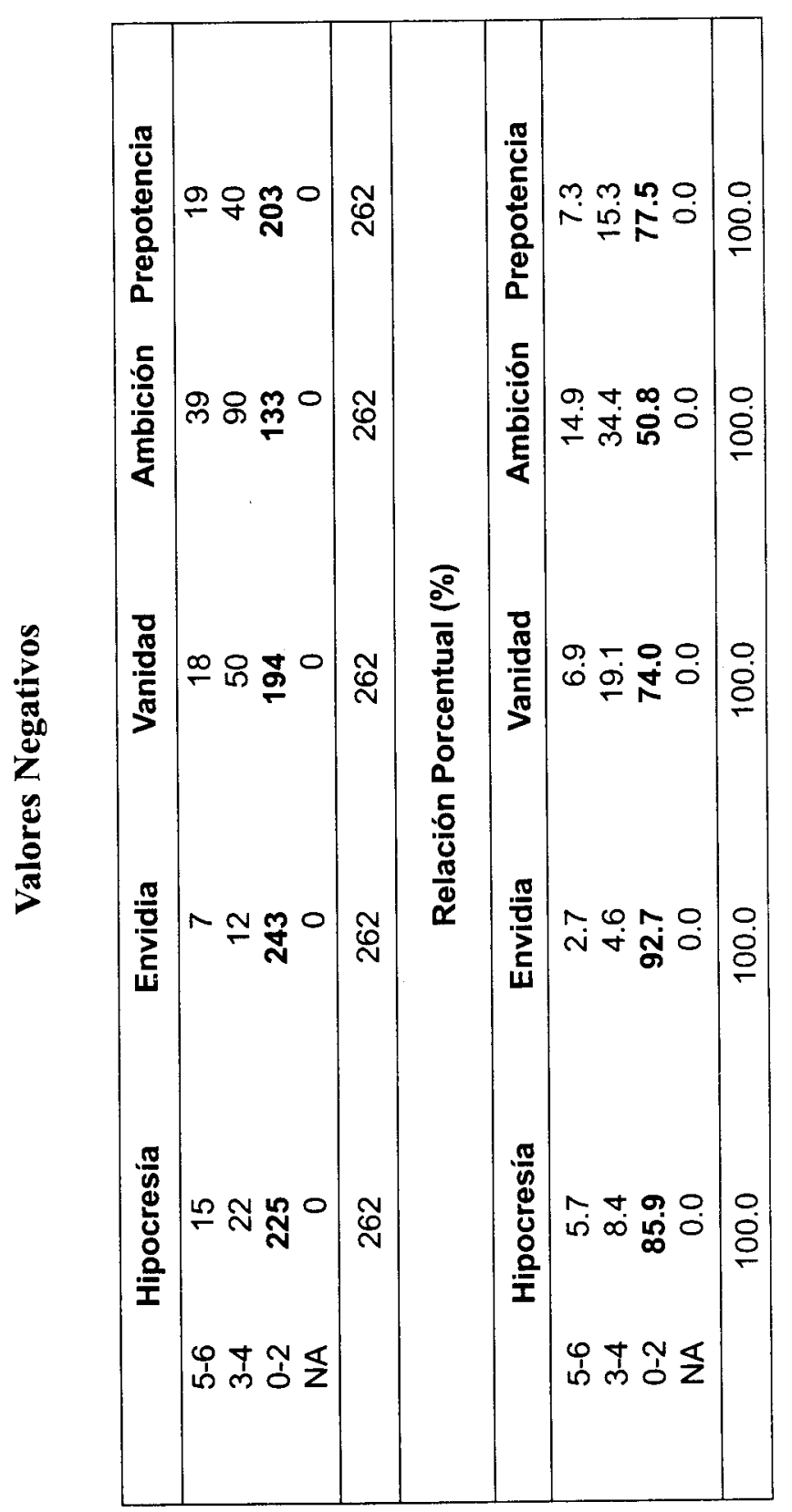




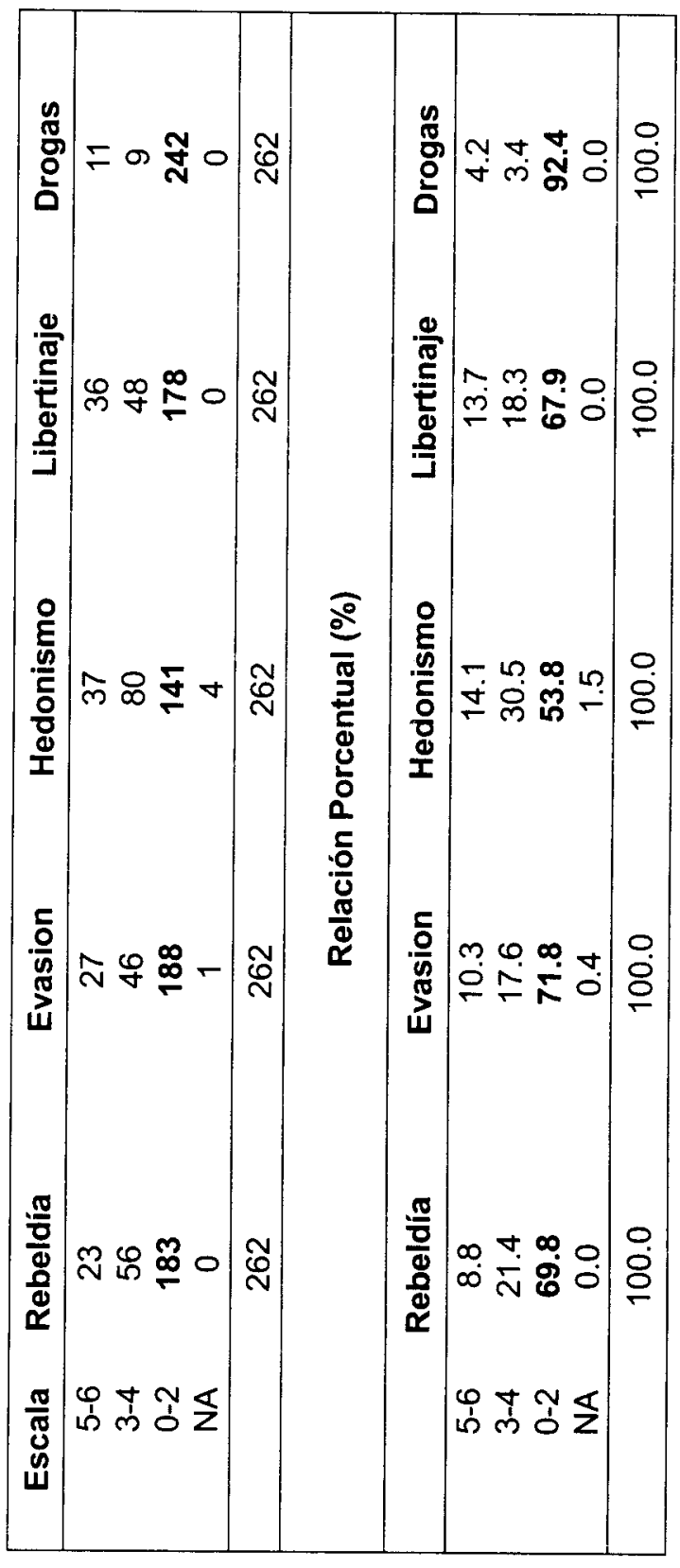


votaciones están ubicadas en lo que para ellos y ellas es lo "nada importante" para sus vidas.

En la medida en que rechazan las drogas o la evasión, hedonismo, libertinaje, etc., como normas que puedan ser importantes o muy importantes para ellos, están claramente refiriéndonos a su identificación con la moralidad del valor que ellos asumen como bueno y estableciendo una relación proporcional en la que valoran lo positivo y rechazan lo negativo.

El propio Frondizi dirá que el sentido de desarmar el valor en su bipolaridad es lo que permite que comprendamos que cada valor tiene esas dos caras, que ambos se encarnan en la sociedad y en cada ambiente con nombre y apellido y que la tarea moral será construir y reforzar el polo positivo mientras también será moral rechazar el polo negativo que era en el fondo lo planteado por Sheler cuando hablaba de "preferir y posponer" con la diferencia de que el presuponía y proponía cuáles eran los positivos y cuáles los negativos y según él todos estaríamos llamados a caer necesariamente dentro de su escala propuesta.

\subsection{Distribución de Frecuencias: Valores Según Sexo}

En esta interpretación según género se verifica el mismo patrón que cuando hicimos el análisis general sin diferenciación de sexo. El dinero fue puesto en "importante" pero el resto de los valores positivos fueron colocados como "muy importantes" tanto en los masculinos como en los femeninos. Como excepción aparece "competencia", "apariencia física", "posición social" y "popularidad" que son valoradas como "muy importantes" en el sexo femenino sobre el masculino.

La moda tiene mayor valoración como "muy importante" en los varones lo mismo que el interés por lo sexual y el placer. En el resto de las valoraciones positivas no hay diferencias significativas en la forma de estimar ni en la forma de ordenar sus valoraciones. 


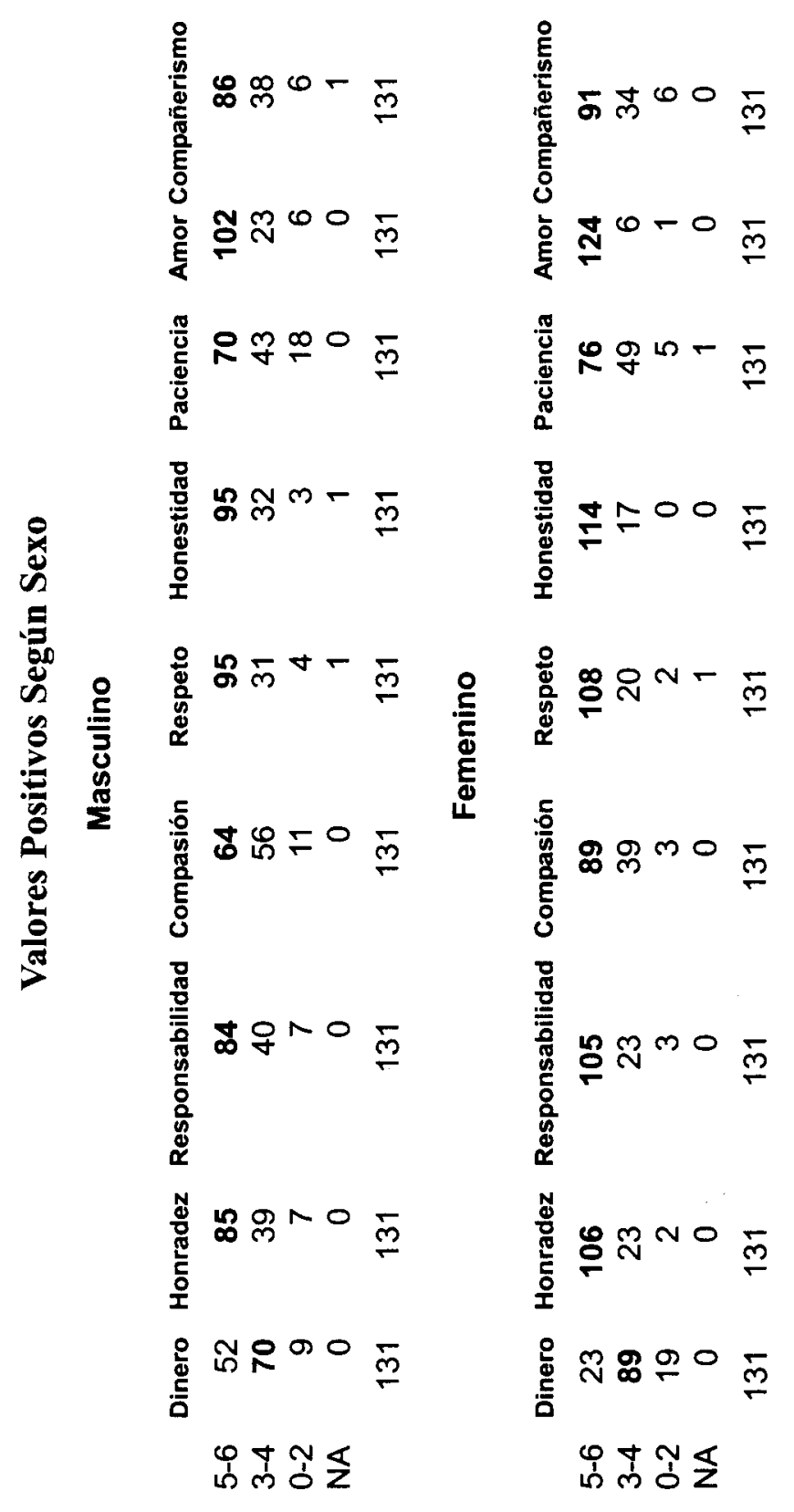

626 


$$
\begin{aligned}
& \text { 焉 }
\end{aligned}
$$

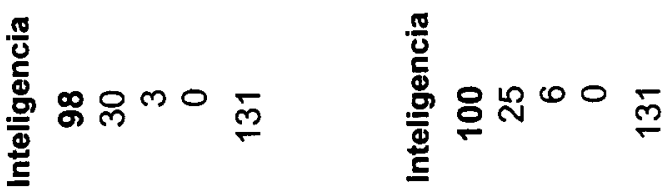

$$
\begin{aligned}
& \text { 覀 }
\end{aligned}
$$

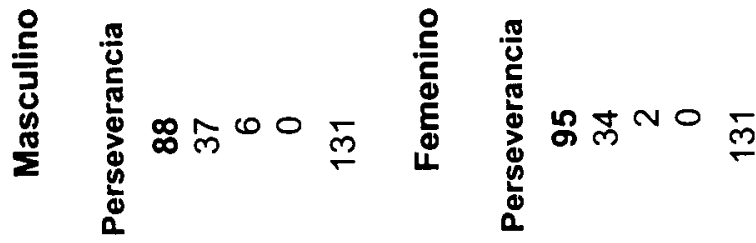

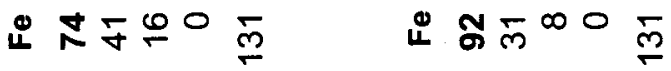

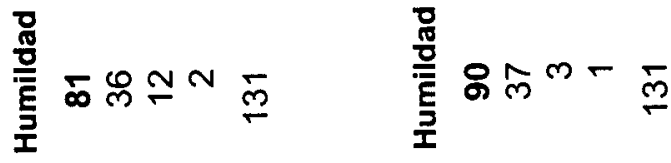

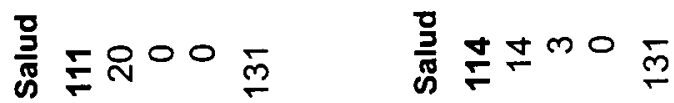

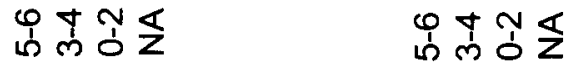




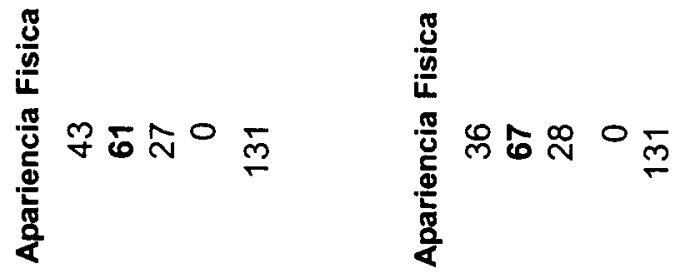

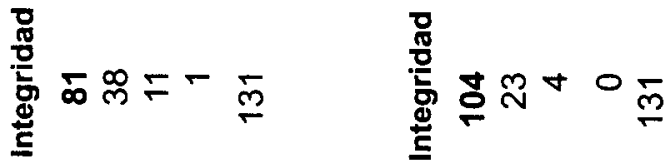

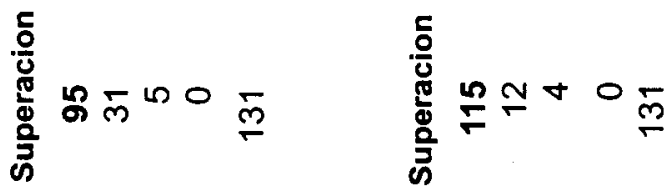

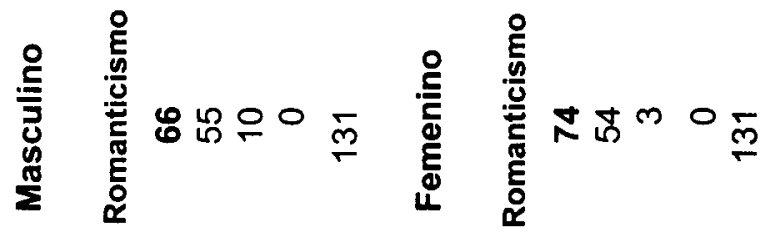

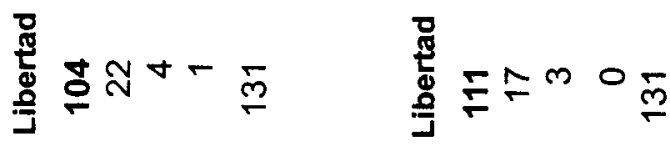

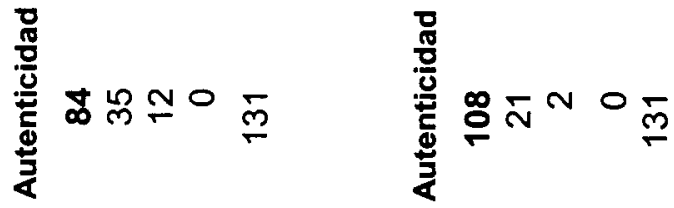

$$
\begin{aligned}
& \text { 递 }
\end{aligned}
$$

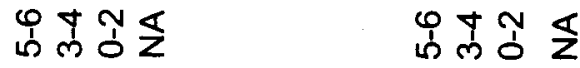




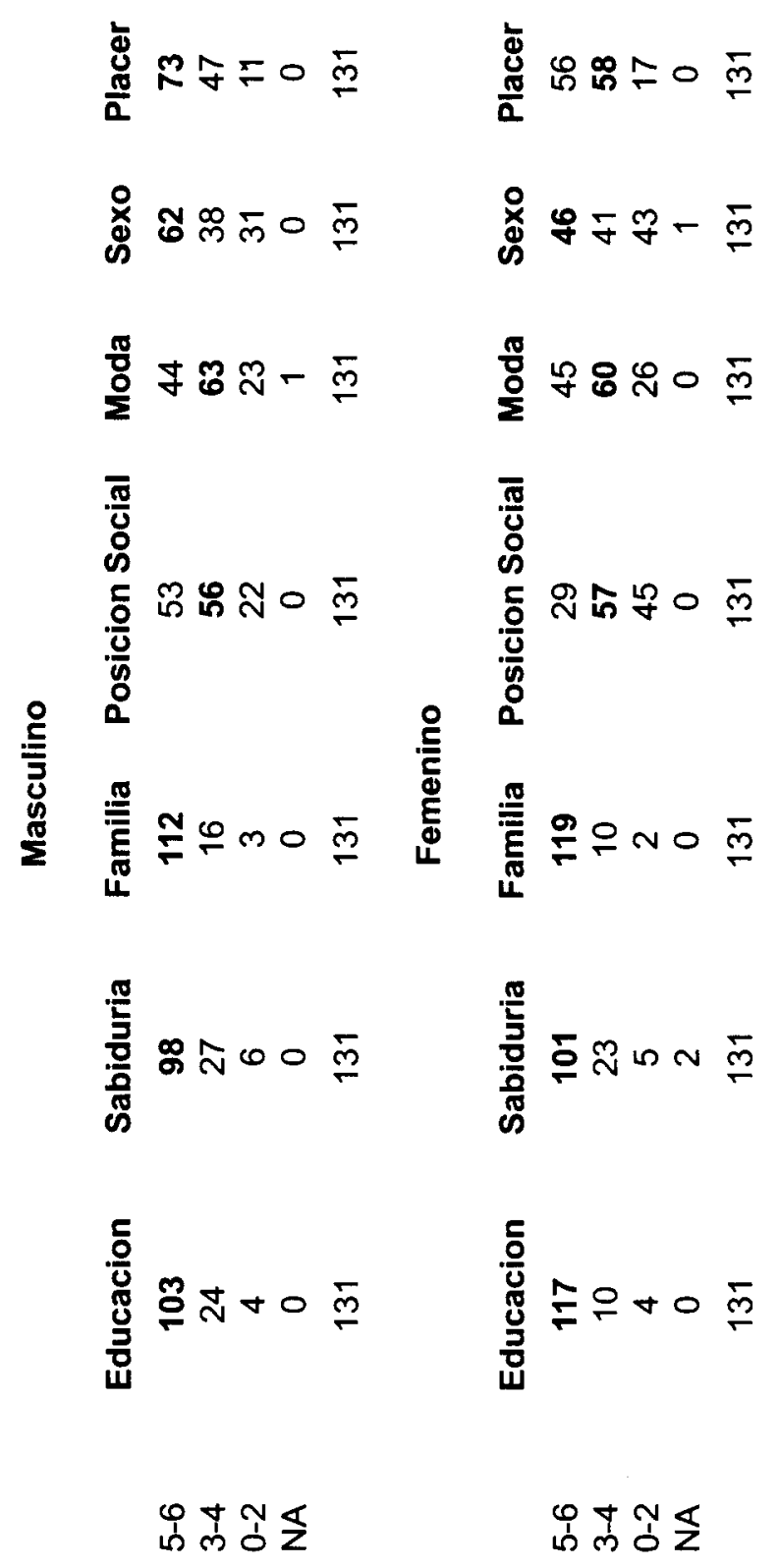

629 


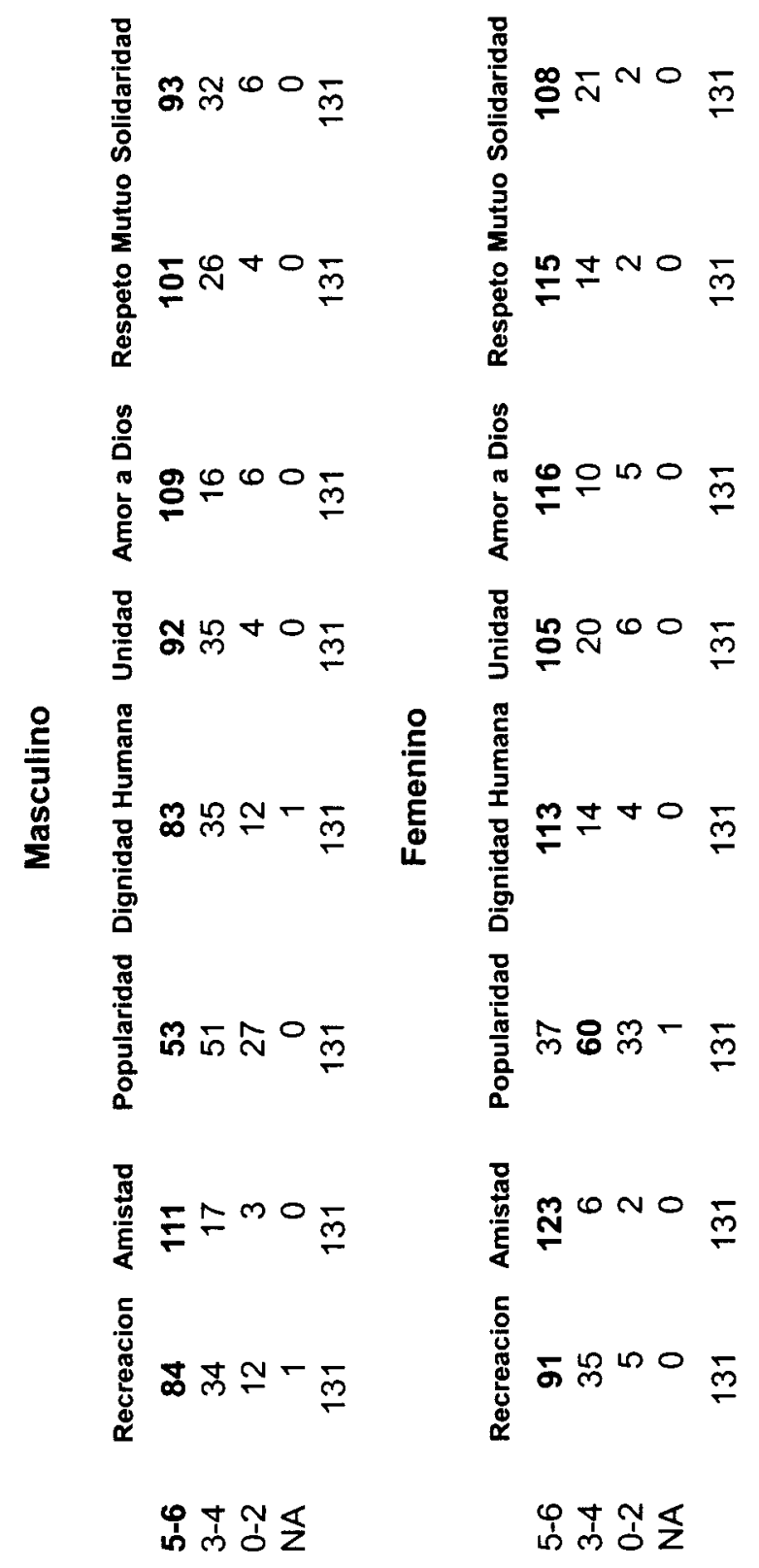

630 
赵

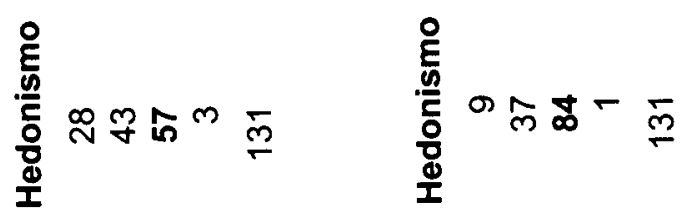

咅 咅

䁹

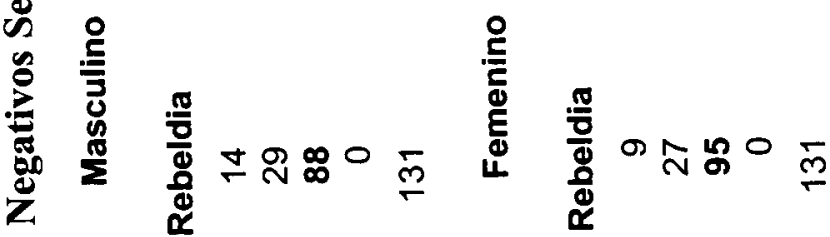

$\frac{\sqrt{3}}{\pi}$

金

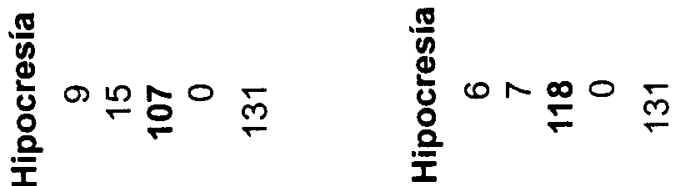

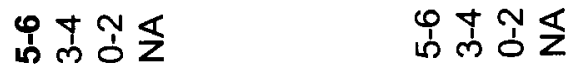




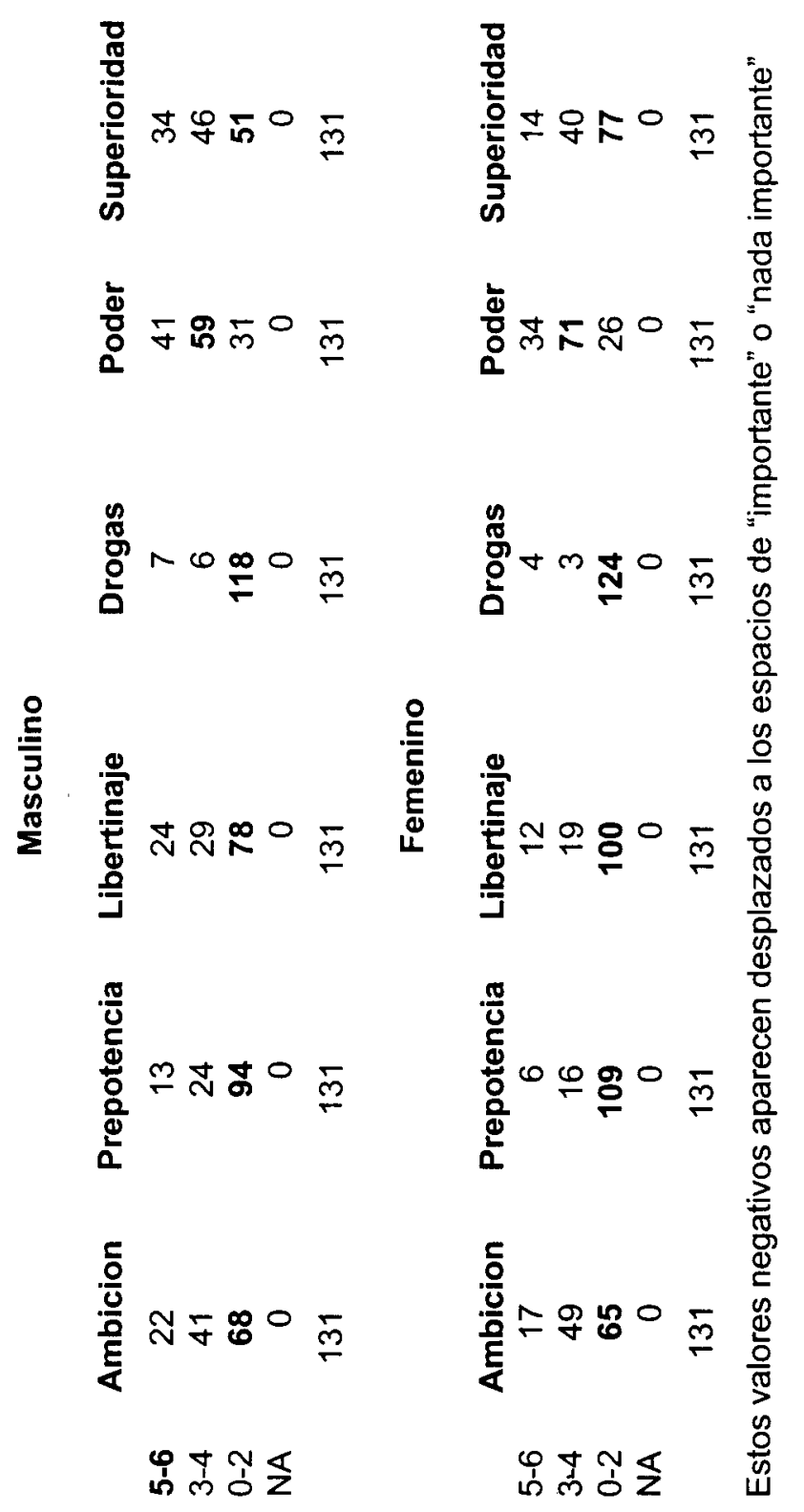




\section{CONCLUSIONES.}

Como hemos señalado la adolescencia media se caracteriza básicamente por el proceso de independencia, de invulnerabili$\mathrm{dad}$, expresiones afectivas y sexuales y reconocimiento del grupo de pares con una pérdida transitoria de los lazos intrafamiliares.

En base a estos intereses se convierte esta etapa en presa fácil para adquirir nuevos modelos, ídolos, patrones y solo la existencia de una base axiológica que provenga desde la niñez y el entorno donde se ha desarrollado ofrecerá garantía de que puedan haber estimaciones positivas en la adolescencia media y en el transcurso de la vida toda.

Los valores se forman a lo largo de toda la vida, pero su escenario preferencial son los espacios pequeños en el largo tiempo, es decir que el hogar es el modelo por excelencia ya que es el lugar más pequeño donde permanecemos por más tiempo reproduciendo valores, asumiéndolos o negándolos.

La escuela es el segundo lugar de importancia con eso criterios, lo cual plantea de manera obvia la necesidad de que estos dos espacios hagan coincidir sus estrategias y esfuerzos para garantizar un espacio moral de construcción de las estimaciones y de las prácticas.

En nuestra investigación aparece un indicador de importancia ya que a pesar de estar hablando de una población pequeña de escuelas, colegios y barrios de clase media, hay una unidad interesante en la forma de estimar y valorar.

Los valores positivos aparecen con una prevalencia marcada sobre los negativos lo que indica que por lo menos en la esfera de las estimaciones los jóvenes están claros en la necesidad de enmarcar en el mundo de la moralidad las valoraciones.

Desde la perspectiva de género no hay diferencias sustantivas que pemitan hacer un análisis más exhaustivos para dar razón de los hallazgos y más bien parecen indicar que no hay estimacio- 
nes propias de los géneros sino que la orientación hacía la búsqueda y hacía la construcción de lo bueno aparece como tendencia.

Nos resta poder trabajar ahora la relación existente entre estimación y comportamiento porque como ya antes señalamos, el valorar positivamente no significa necesariamente que se actúe en consecuencia.

Los valores generales nos demuestran una inclinación favorable hacía el valor moral y lo que hace es plantearnos retos para nuevas investigaciones.

\section{BIBLIOGRAFÍA}

1. Aristóteles. Retórica II 12:1389

2. Blanco, Juan Antonio. Tercer Milenio. Edición del Centro Félix Valera, La Habana, Cuba, 1998.

3. Bolivar, Antonio. La evaluación de valores y actitudes, Grupo ANAYA, S.A., Madrid, España, 1998.

4. Cairo, Valeirón y Zaiter. «Sociedad, Cultura e Identidad en la República Dominicana». Tesis de maestria en sicologia comunitaria, UASD, Santo Domingo 1998.

5. Cortina, Adela. El mundo de los valores. Editorial Codice ltda, Bogotá.2000.

6. De Láncer, Víctor Hugo. "Sistema Educación y Cultura Democrática". Periódico El Siglo, Domingo, 22/10/2000

7. Delval, Juan; Enesco, Ileana. Hacer Reforma Moral, Desarrollo y Educación. Grupo Anaya. S.A, Madrid. 1998

8. Donas, S. "Marco epidemiológico conceptual de la salud integral del adolescente" Versión revisada y ampliada, noviembre 1997, OPS. Costa Rica, . 1997.

9. Dulanto, Enrique. "El adolescente". Asociación Mexicana de Pediatría. Educación en Valores A.C., Mexico, 1989

10. Edisa. «Educación a distancia en salud del adolescente. Módulo 9».La educación en la Europa de los siglos XVIII y XIX.Fundación Kellogg, Buenos Aires, 1997 
11. Secretaría de Estado de Educación y Cultura. Educación en Valores. Editora Centenario, S.A., Santo Domingo, Abril 1998. 112pp.

12. F. Compagnoni, G. Piana y S. Privitera, M. Vidal. Nuevo Diccionario de Teología Moral, Ediciones Paulinas, Madrid, España, 1992.

13. Frondizi, R. ¿Qué son los valores?, FCE, $5^{\text {ta }}$. Edición. México, D.F. 1972.

14. Secretaría de Estado de Educación Bellas Artes y Cultos. Fundamentos del Curriculum (Tomo Iy II), Editora Alfa \& Omega, 1. ${ }^{\text {era }}$. Edición, Santo Domingo, 1994.

15. Gafo, Javier. "Los principios de justicia y solidaridad en bioética", en: Cuadernos Regional de Bioética, edición N. ${ }^{\circ}$ 6, agosto 1998, Santiago, Chile.

16. González Alvarez, Luis José. Etica Latinoamericana, Universidad Santo Tomás-USTA, Bogotá, Colombia, 1994.

17. Gracia, Diego. Notas de Cátedra Magister Bioética. Universidad de Chile, Chile, 1998.

18. Gracia, Diego. Fundamentos de Bioética, Eudema, S.A., Madrid, España, 1987,

19. Gracia Diego. «Etica de los Confines de la Vida». Ética y Vida, N ${ }^{\circ} 3$ .El Buho. Colombia. 1998

20. Hernández Sampieri, Roberto; Fernández Collado, Carlos; Baptista Lucio, Pilar. Metodología de la Investigación. Editora McGraw-Hill, S.A. México, D.F., 1998. 